\title{
Dynamic dead space in face masks used with noninvasive ventilators: a lung model study
}

\author{
E. Saatci*, D.M. Miller", I.M. Stell ${ }^{\top}$, K.C. Lee*, J. Moxham
}

Dynamic dead space in face masks used with noninvasive ventilators: a lung model study. E. Saatci, D. M. Miller, I.M. Stell, K.C. Lee, J. Moxham. (C)ERS Journals Ltd 2004.

ABSTRACT: The aim of this study was to determine what the influence of different designs of face masks and different noninvasive ventilator modes would be upon total dynamic dead space.

Using a spontaneous breathing model, total dynamic dead space was measured when using 19 commercially available face masks and a range of ventilators in various ventilation modes.

Total dynamic dead space during spontaneous ventilation was increased above physiological dead space from $32 \%$ to $42 \%$ of tidal volume by using face masks. The use of noninvasive ventilation modes such as bilevel and continuous positive airway pressure, with continuous pressure throughout the expiratory phase, reduced total dynamic dead space to approach physiological dead space with most face masks. Pressure assist and pressure support ventilation decreased total dynamic dead space to a lesser degree, from $42 \%$ to $39 \%$ of tidal volume. Face masks with expiratory ports over the nasal bridge resulted in beneficial flow characteristics within the face mask and nasal cavity, so as to decrease total dynamic dead space to less than physiological dead space from $42 \%$ to $28.5 \%$ of tidal volume.

Exhaust ports over the nasal bridge in face masks effect important decreases in dynamic dead space provided positive pressure throughout the expiratory phase is used. Eur Respir J 2004; 23: 129-135.
*Division of Engineering, King's College London, ${ }^{\text {\#}}$ Dept of Anaesthetics, Guy's, King's and St Thomas' School of Medicine, Guy's Hospital, and 'Dept of Respiratory Medicine, King's College Hospital, London, UK.

Correspondence: D.M. Miller, Dept of Anaesthetics, Guy's, Kings and St. Thomas' School of Medicine, Guy's Hospital, London SE1 9RT, UK.

Fax: 442079554050

E-mail: donald.miller@kcl.ac.uk

Keywords: Dynamic dead space, face masks, lung model, noninvasive ventilators

Received: April 82003

Accepted after revision: July 232003

This study was supported by a grant from the Guy's and St Thomas' Hospital Charitable Foundation, and the Dept of Health National Health Service New and Emerging Applications of Technology Programme.
One of the main goals in noninvasive ventilation (NIV) of chronic obstructive pulmonary disease (COPD) is to improve gas exchange. However, unavoidable physiological dead space ( $V \mathrm{D}$,phys) and dynamic apparatus dead space ( $V \mathrm{D}, \mathrm{ap})$ comprising alveolar gases that are inhaled a second time into the alveolar compartment, encroach on alveolar ventilation. This influences pulmonary mechanics and breathing pattern [1-4]. Flow through face masks appears to have significant impact upon dynamic dead space (VD) [5]. $V \mathrm{D}$ has already been well defined [6]. Factors such as flow rate and flow pattern influence the position of the interface between air and alveolar gas. Thus, $V \mathrm{D}$, phys is influenced by inspiratory flow rate, expiratory flow rate and tidal volume $(V \mathrm{~T})$ [6-8]. As $V \mathrm{D}$,phys changes with $V \mathrm{~T}$, it makes good sense to express it as a fraction of resting or ventilated $V \mathrm{~T}(V \mathrm{D}$,phys $/ V \mathrm{~T})$.

Different measurement methods may be applied to measure $V D$,phys in clinical practice. They include the standard reference technique "single-breath carbon dioxide" of FLETCHER et al. [6], "multiple-breath nitrogen" of ARBORELIUS et al. [9], "oscillating inspired argon" of WiLLIAMs et al. [10], and "oscillating inspired oxygen" of WiLLIAMs et al. [11]. Although determination of $V \mathrm{D}$, phys is well studied in the literature, there are no studies that measure $V D$,ap in NIV. However, the use of face masks has the potential to influence $V \mathrm{D}$,ap [1]. In this study, $V \mathrm{D}$,ap is defined as the $V \mathrm{D}$ that exceeds $V \mathrm{D}$, phys. The factors that influence the value of $V \mathrm{D}$, ap comprise the interface between ventilating apparatus and the

For editorial comments see page 7. face of the patient including flow patterns and pressure waveforms. Although some manufacturers provide static volume measurements within face masks, the great variety of face masks combined with the different ventilator modes is likely to influence $V \mathrm{D}$ in ways not yet defined. Furthermore, different ventilators with similar ventilation modes may have differing effects upon $V \mathrm{D}$. Thus, the aim of this study is to determine the influence of different designs of face masks and different noninvasive ventilator modes upon total dynamic dead space $(V \mathrm{D} / V \mathrm{~T})$. A lung model was developed to measure the dynamic value of total dead space $(V \mathrm{D}), V \mathrm{D}$, phys $+V \mathrm{D}$, ap, in terms of the fraction $V \mathrm{D} / V \mathrm{~T}$ for all commercially available NIV face masks with various ventilation modes.

\section{Material and methods}

\section{Study design}

In this study, a previously designed lung model was modified (fig. 1). Since the bellows, B1 and B2 appear to occupy the position of the lungs in figure 1 , do not make the mistake of trying to understand the model as being an attempt at mimicking physiological lungs. The principle of the lung model design has been reported previously [12]. The lung model is designed to test the performance of equipment with the specific aim of measuring $V \mathrm{D} / V \mathrm{~T}$ relating to the equipment under the expected clinical conditions. It is not designed to mimic any lung conditions or lung physiology. Therein is its 


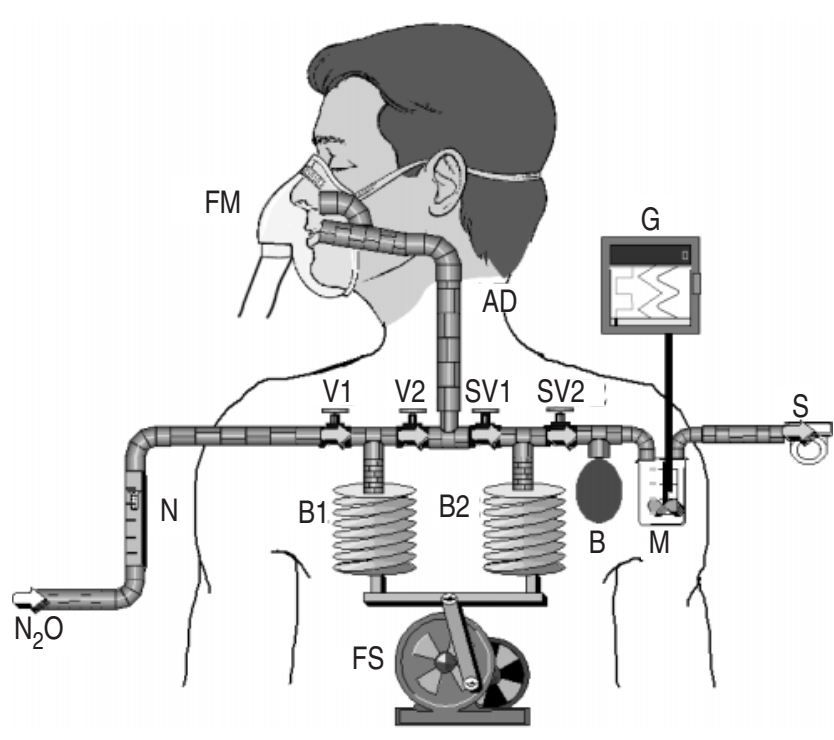

Fig. 1.-Model lung. FM: noninvasive ventilation (NIV) face mask; AD: anatomical dead space; G: gas monitor; V1, V2: one-way valves; SV1, SV2: solenoid valves; $\mathrm{S}$ : scavenging system; $\mathrm{N}_{2} \mathrm{O}: 100 \%$ nitrous oxide; $\mathrm{N}$ : $\mathrm{N}_{2} \mathrm{O}$ flow-meter tube; $\mathrm{B} 1, \mathrm{~B} 2$ : bellows; $\mathrm{B}$ : 1-L bag; M: mixing chamber and fan; FS: Frank-Starling pump.

particular advantage; it has a targeted objective that is not contaminated by any clinical condition. In essence, the lung model provides $V$ Ts of expiratory gas $(100 \%$ nitrous oxide $\left(\mathrm{N}_{2} \mathrm{O}\right)$ representing alveolar gas) expelled into a NIV face mask ventilation system. The same $V \mathrm{~T}$ of the mixture that results within the face mask is then inhaled by the model. Since the gas in the atmosphere or from a noninvasive ventilator contains zero $\mathrm{N}_{2} \mathrm{O}$, the concentration of $\mathrm{N}_{2} \mathrm{O}$ that is inhaled by the model will be a precise measurement of that portion of the volume of exhaled alveolar gas that is rebreathed.

Briefly, a mechanical test lung (Michigan Instruments Inc., Grand Rapids, MI, USA) has both bellows linked so that they move together by a motor-driven Starling pump arrangement. The Starling pump sinusoidally and equally moves both compartments of the test lung in order to simulate spontaneous breathing. $V \mathrm{~T}$ and respiratory rate can be precisely regulated by means of a stroke-control mechanism and a gearbox.

Solenoid valves (SMC Pneumatics Ltd, Milton Keynes, UK) and one-way valves (Hans Rudolph Inc., Kansas City, MO, USA) were used to separate expiratory $\left(100 \% \quad \mathrm{~N}_{2} \mathrm{O}\right)$ from the inspiratory (mixture of $\mathrm{N}_{2} \mathrm{O}$ and air) gases. $100 \%$ $\mathrm{N}_{2} \mathrm{O}$ was supplied via a flow meter to the expiratory side of the model. During inspiration, while the bellows B1 fill with $\mathrm{N}_{2} \mathrm{O}$ through a one-way valve $\mathrm{V} 1$, the bellows $\mathrm{B} 2$ aspirate inspiratory gases through an electronically controlled open solenoid valve SV1. During expiration, the one-way valve V2 allows the bellows B1 to empty through to the trachea AD of the model, while solenoid valve SV1 is closed. At the same time, the bellows B2 force the air mixture to the sampling apparatus via the open solenoid valve SV2.

Solenoid valves were used in the model because inspiratory positive airway pressure (IPAP) creates a positive pressure in B2 at the end of inspiration, with the potential of generating unwanted gas flow to the sampling side if passive one-way valves alone are used. The discharge of the inhaled gas mixture to the sampling apparatus is thereby limited to the expiratory phase alone. This is achieved by the switch arrangement mounted on the shaft of the Starling pump to trigger inspiratory and expiratory phases. The computer and data acquisition system (Labview; National Instruments Inc.,
Austin, TX, USA) collects switch signals and controls solenoid valves accordingly. The Resusci Anne Laerdal mannequin head (Laerdal Medical Corp., Stavanger, Norway) was modified by removing the airway obstruction mechanism and a suitable corrugated tube (anatomical dead space) was inserted for attachment to the lung model as shown in figure 1.

To facilitate reliable sampling, the intermittent $V$ Ts expelled through SV2 were converted to a more steady flow by the interposition of a $1-\mathrm{L}$ reservoir bag $\mathrm{B}$. In addition, the reservoir bag contributed to mixing, which was completed in a chamber containing a fan. A computer fan was placed inside of a 2-L chamber for mixing the gases. The computer fan had an axial flow impeller and, when the agitator was centrally mounted, excellent top-to-bottom motion was produced resulting in good mixing. $\mathrm{N}_{2} \mathrm{O}$ concentration was measured using a gas monitor (Datex-Ohmeda, Ultima; Division Instrumentarium Corp., Helsinki, Finland) and the results stored in the computer using the data acquisition system.

Dead space of the system (mannequin head and trachea) was measured and adjusted to achieve a $V \mathrm{D}$,phys $/ V \mathrm{~T}$ of 0.32 $[13]$ to mimic the expected $V \mathrm{D}$, phys in patients. $V \mathrm{D}$, phys was therefore $141 \mathrm{~mL}$, since the $V \mathrm{~T}$ was $440 \mathrm{~mL}$. In this model, the fractional concentration of $\mathrm{N}_{2} \mathrm{O}$ is equal to $V \mathrm{D} / V \mathrm{~T}$. $V \mathrm{~T}$ was measured using a calibrated pitot-tube flow sensor (Dlite; Datex-Ohmeda Ultima) inserted between B2 and SV2. $\mathrm{N}_{2} \mathrm{O}$ sensing was calibrated with $100 \% \mathrm{~N}_{2} \mathrm{O}$ and an $\mathrm{N}_{2} \mathrm{O}$ calibration mixture $(40 \%)$ supplied by Datex-Ohmeda. The stroke of the pump was adjusted to achieve a spontaneous $V \mathrm{~T}$ of $440 \mathrm{~mL}$. Face masks were tested in random order with the operator blinded to the results during the test.

Of the lung models that have been used for measuring $V D$, this is the only one that provides a direct value for $V \mathrm{D} / V \mathrm{~T}$ i.e. equal to the fractional concentration of $\mathrm{N}_{2} \mathrm{O}$. The respective use of a very large dead space, one that exceeded the $V$ T many times, and zero dead space gave $\mathrm{N}_{2} \mathrm{O}$ values of $100 \%$ and $0 \%$. In spontaneous ventilation, when the pressure in the system is low, solenoid valves are not required as the pressure changes in the apparatus ensure precise opening and closing. However, the use of positive pressure with different ventilation modes could cause gas to flow through SV2 and into the sampling system at an unwanted time during the respiratory cycle. This would result in a value for $V D$ that is excessively large if the timing of the solenoid valves were imprecise. In order to check that the solenoid valves were performing according to the expected reliability of the model, the results obtained using positive pressure with identical apparatus were compared and found to be similar with those obtained in spontaneous ventilation.

\section{Static volume of face masks}

To enable us to examine the relationship between the static and dynamic volumes within face masks, the static volumes of the space between the face of a mannequin and face masks were measured by two different methods. The space was filled with fine dry granular salt and a soft pliable substance (dough) of known constant density of $1.41 \mathrm{~g} \cdot \mathrm{mL}^{-1}$ in all of 18 NIV face masks and one total face mask (fig. 2). The recorded values were the mean of four measurements.

\section{Spontaneous breathing}

Eighteen NIV face masks and one total face mask were tested on the mannequin head (table 1). The study was conducted utilising the face masks in the prescribed manner, 


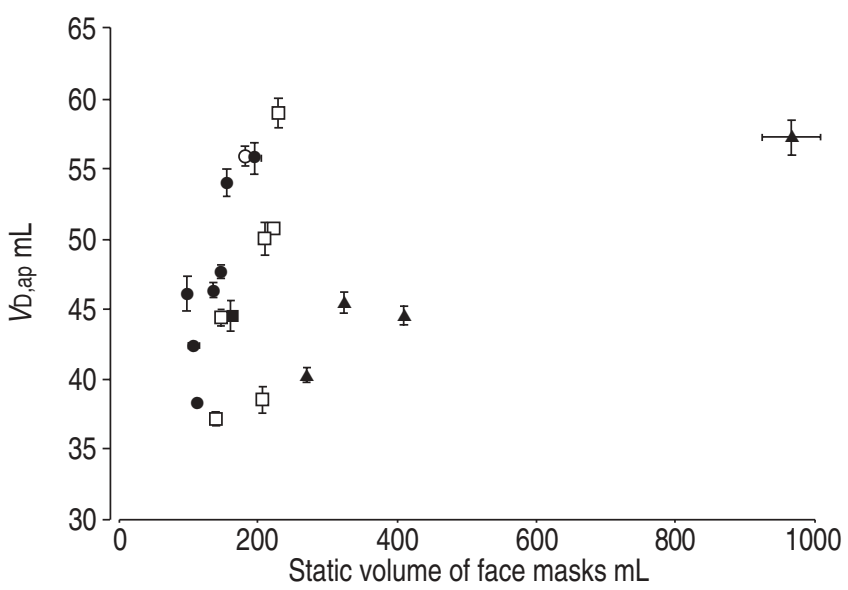

Fig. 2.-Dynamic apparatus dead space ( $V \mathrm{D}$,ap) of facemasks versus their static volumes (Resusci Anne Laerdal mannequin head). 0 : B\&D Electromedical face mask; $\bigcirc$ : Koo face mask; $\mathbf{\square}$ : Hans Rudolph facemask; $\square$ : ResMed face mask; $\boldsymbol{\Delta}$ : Respironics face mask.

i.e. no occlusion of holes on face masks. Face masks were applied in a manner sufficient to prevent any leak during breathing in order to obtain the largest $V D$ values. Lung model settings were 19 breaths $\cdot \mathrm{min}^{-1}$ and $440 \mathrm{~mL} V \mathrm{~T}$. Each face mask was applied for $5 \mathrm{~min}$ to the mannequin head and the $\mathrm{N}_{2} \mathrm{O}$ concentration recorded. This value was based upon the last $90 \mathrm{~s}$ of the $5 \mathrm{~min}$ of recorded values sampled at 30 samples $\cdot \mathrm{s}^{-1}$, these being the most stable. They included 2,700 readings from which mean value and SE were calculated.

\section{Noninvasive ventilation}

To assess the effects of different noninvasive ventilators, five commonly available machines were used (table 2). The masks were connected to the ventilators via their recommended patient circuits (fig. 3). This included corrugated tubes and connectors with exhaust ports for Puritan Bennett Knightstar 335 (Puritan Bennett, Pleasanton, CA, USA), Respironics BIPAP S/T (Respironics Inc., Murrysville, PA, USA) and ResMed Sullivan VPAP S/T (ResMed Ltd, North Ryde, New South Wales, Australia). For the Breas 401 (Breas Medical AB, Mölnlycke, Sweden) and NIPPY 1 (B\&D Electromedical, Stratford upon Avon, UK), this included corrugated tubes, exhaust valves, exhalation valve lines and pressure lines. Spontaneous breathing patterns remained constant throughout. All ventilators were set to their most sensitive triggering level for both inspiration and expiration, where applicable. Puritan Bennett Knightstar 335 was used both bilevel and continuous positive airway pressure (CPAP) mode.

\section{Results}

Figure 2 shows $V \mathrm{D}$, ap of face masks compared with static volume measurements when the face masks were applied to a Resusci Anne Laerdal mannequin head.

Figure 4 shows the $V \mathrm{D} / V \mathrm{~T}$ fractions in spontaneous breathing with all of the face masks. All face masks increased the $V \mathrm{D}$ from 0.32 to 0.42 , an average increase of $33.82 \%$.

The use of NIV modes, such as Bilevel (fig. 5) and CPAP

Table 1.-Noninvasive ventilation face masks

\begin{tabular}{|c|c|c|c|c|}
\hline Company name & Mask name & Size & Abbreviation used & Position of exhaust ports on face masks \\
\hline B\&D Electromedical & Aircraft & M & BD Air & 0 \\
\hline B\&D Electromedical & Hebden & $\mathrm{S}$ & BD HS & 0 \\
\hline B\&D Electromedical & Hebden & M & BD HM & 0 \\
\hline B\&D Electromedical & Hebden & $\mathrm{L}$ & BD HL & 0 \\
\hline B\&D Electromedical & Fleximask & $\mathrm{S}$ & $\mathrm{BD} F \mathrm{~F}$ & 0 \\
\hline B\&D Electromedical & Fleximask & M & BD FM & 0 \\
\hline B\&D Electromedical & Fleximask & $\mathrm{L}$ & BD FL & 0 \\
\hline Hans Rudolph & Full face mask & & HR FM & 1 (cheek) \\
\hline Koo & Full face mask system & & KOO FM & 0 \\
\hline ResMed & Mirage NV full face mask & $\mathrm{S}$ & RM NS & 0 \\
\hline ResMed & Mirage NV full face mask & $\mathrm{M}$ & RM NM & 0 \\
\hline ResMed & Mirage NV full face mask & $\mathrm{L}$ & RM NL & 0 \\
\hline ResMed & Full face series 2 & $\mathrm{~S}$ & RM SS & Series (nasal bridge) \\
\hline ResMed & Full face series 2 & M & RM SM & Series (nasal bridge) \\
\hline ResMed & Full face series 2 & $\mathrm{~L}$ & RM SL & Series (nasal bridge) \\
\hline Respironics & Spectrum & $\mathrm{S}$ & RS SS & 1 (connector) \\
\hline Respironics & Spectrum & M & RS SM & 1 (connector) \\
\hline Respironics & Spectrum & $\mathrm{L}$ & RS SL & 1 (connector) \\
\hline Respironics & Total face mask & & RS TFM & 2 (cheeks) \\
\hline
\end{tabular}

S: small; M: medium; L: large.

Table 2. - Noninvasive ventilators and settings

\begin{tabular}{lcccccc}
\hline NIV ventilators & Respironics & Breas 401 & NIPPY 1 & $\begin{array}{c}\text { Puritan Bennett } \\
335 \text { (Bilevel) }\end{array}$ & $\begin{array}{c}\text { Puritan Bennett } \\
\text { 335 (CPAP) }\end{array}$ & $\begin{array}{c}\text { ResMed Sullivan } \\
\text { VPAP S/T }\end{array}$ \\
\hline Ventilation mode & Bilevel & Pressure support & Pressure assist & Bilevel & CPAP \\
IPAP & 16 & 16 & 16 & 16 & 5 & Bilevel \\
EPAP & 4 & 0 & 0 & 4 & 5 & 16 \\
\hline
\end{tabular}

NIV: noninvasive ventilation; CPAP: continuous positive airway pressure; BIPAP: bilevel positive airway pressure; IPAP: inspiratory positive airway pressure; EPAP: expiratory positive airway pressure. 
a)

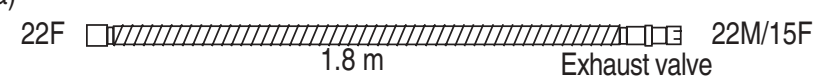

b)

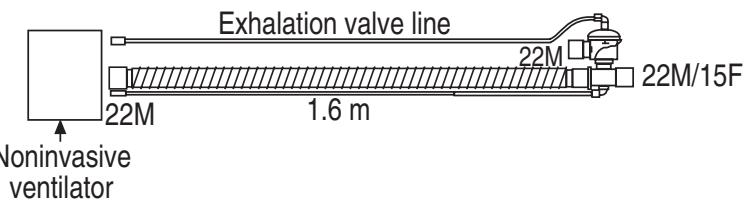

Fig. 3.-Recommended breathing systems for the different ventilators used in this study. a) Corrugated tube and connector with exhaust port for Puritan Bennett Knightstar 335, Respironics BIPAP S/T and ResMed Sullivan VPAP S/T. b) Corrugated tube, exhaust valve, exhalation valve line and pressure line for Breas 401 and NIPPY 1. Reproduced with permission from Intersurgical Ltd, Wokingham, UK.

(fig. 6), with continuous pressure throughout the expiratory phase, reduced $V \mathrm{D} / V \mathrm{~T}$ to mean values of 0.33 and 0.34 with most face masks, a value that approximates $V D$, phys. Some face mask arrangements resulted in beneficial flow characteristics to decrease $V \mathrm{D} / V \mathrm{~T}$ to less than $V \mathrm{D}$,phys (fig. 5; ResMed full face series 2). Face masks with expiratory ports over the nasal bridge resulted in beneficial flow characteristics within the face mask and nasal cavity, to decrease $V \mathrm{D} / V \mathrm{~T}$ to less than $V \mathrm{D}$,phys from $42 \%$ to $28.5 \%$ of $V \mathrm{~T}$. Exhaust ports at other sites were not nearly as beneficial with ports located in the face mask over the cheeks, performing slightly better than exhaust ports located at the mask-connector site.

Pressure-controlled and pressure-support ventilation decreased $V \mathrm{D} / V \mathrm{~T}$ to a lesser degree, to 0.39 , an average increase of $22.8 \%(0.6)$ above $V D$,phys.

The Respironics BIPAP S/T with IPAP $16 \mathrm{cmH}_{2} \mathrm{O}$ gave values for $V \mathrm{D} / V \mathrm{~T}$ that varied between $0.26-0.39$, (the respective face masks included the ResMed full face series 2 (size small) and the Koo full face mask system; fig. 5).

The Breas 401 pressure-support ventilator and NIPPY 1 pressure-assist ventilator gave almost identical values for $V \mathrm{D} /$ $V \mathrm{~T}$ that varied between $0.34-0.44$ (the respective face masks used were Respironics Spectrum (size small) and Respironics total face mask) for Breas 401, and between 0.33-0.44 (the respective face masks used were B\&D Electromedical Hebden (size small) and Respironics total face mask) for NIPPY 1 (fig. 7).

With the Puritan Bennett Knightstar 335 (bilevel mode),

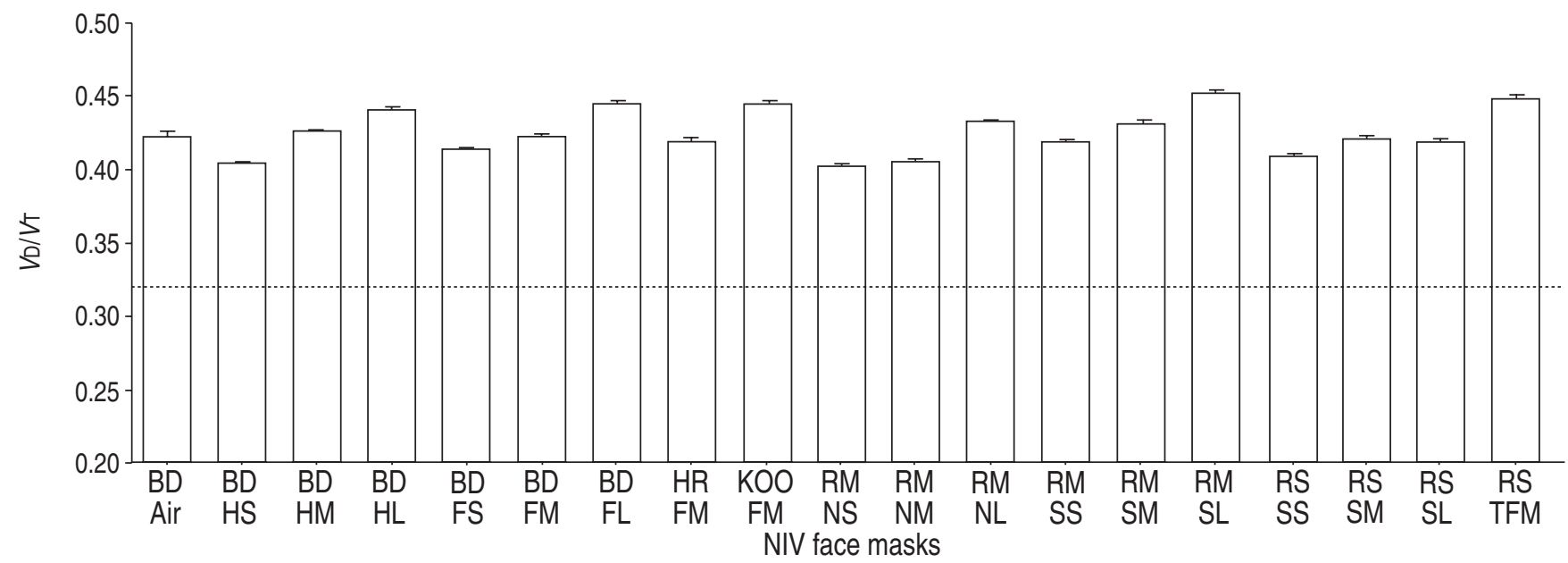

Fig. 4. - Total dynamic dead space $\left(V \mathrm{D} / V_{\mathrm{T}}\right)$ of noninvasive ventilation (NIV) face masks in spontaneous breathing. Refer to table 1 for abbreviations.

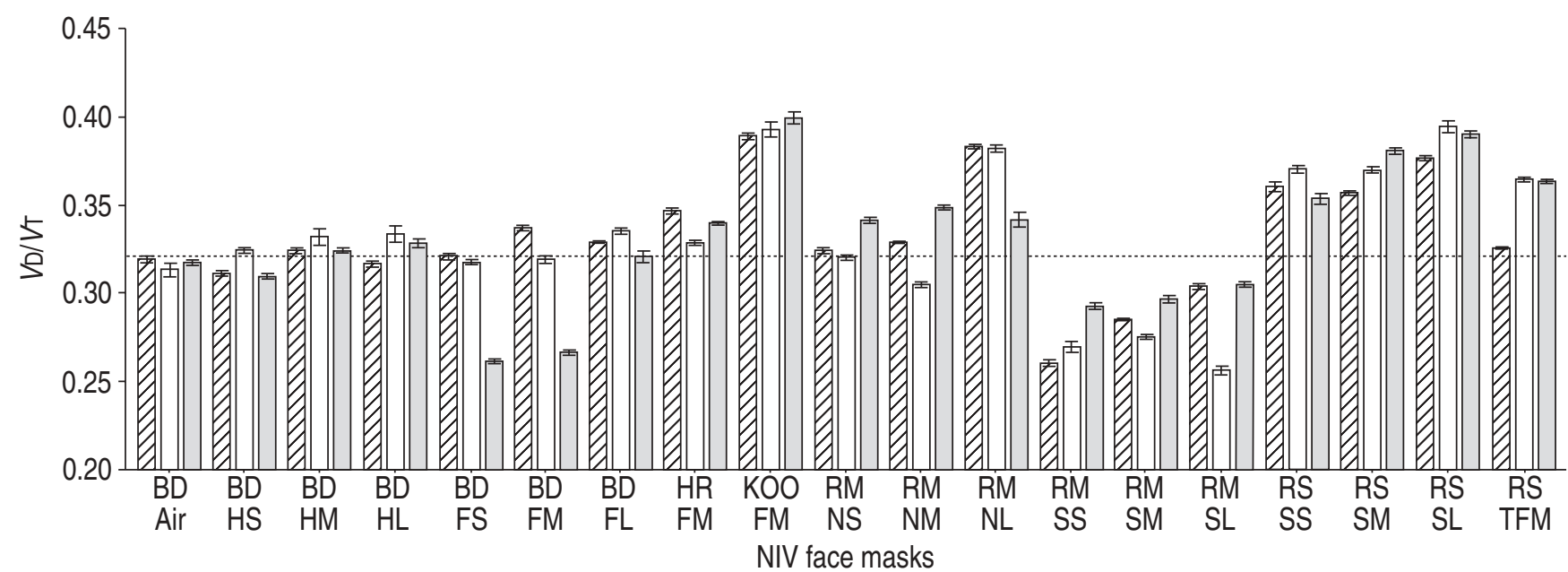

Fig. 5. - Total dynamic dead space $(V \mathrm{D} / V \mathrm{~T})$ of bilevel noninvasive ventilation (NIV) face masks. $\mathbb{Z}$ : Respironics BIPAP S/T; $\square$ : Puritan Bennett Knightstar 335 Bilevel; $\square$ : ResMed Sullivan VPAP S/T. Refer to table 1 for abbreviations. 


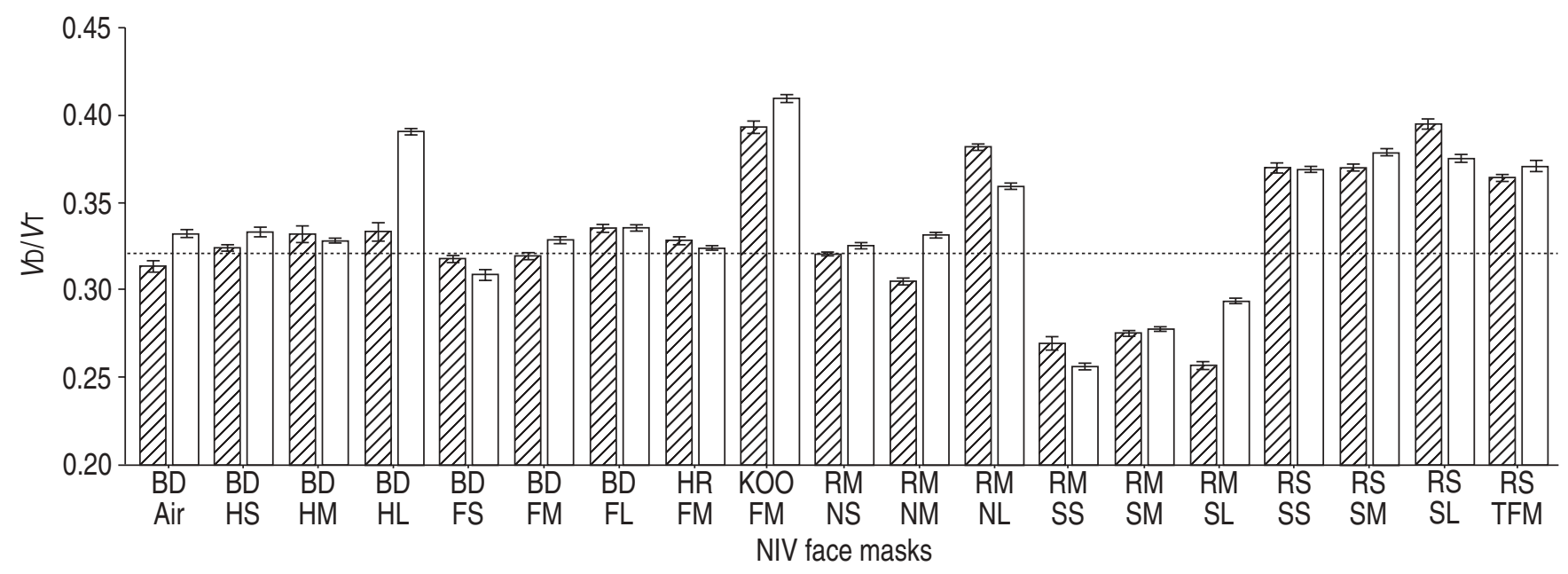

Fig. 6. - Total dynamic dead space $(V \mathrm{D} / V \mathrm{~T})$ in bilevel versus continuous positive airway pressure (CPAP) noninvasive ventilation (NIV) face masks. $\mathbb{Z}$ : Puritan Bennett Knightstar 335 Bilevel; $\square$ : Puritan Bennett Knightstar 335 CPAP. Refer to table 1 for abbreviations.

the values for $V \mathrm{D} / V \mathrm{~T}$ varied between $0.26-0.39$ (the respective face masks used were the ResMed full face series 2 (size large) and Koo full face mask system; fig. 5). When Puritan Bennett Knightstar 335 was used in CPAP mode, the values for $V \mathrm{D} / V \mathrm{~T}$ were no different from its use in Bilevel mode (fig. 6).

With the ResMed Sullivan VPAP S/T the values for $V \mathrm{D} / V \mathrm{~T}$ varied between $0.26-0.40$ (the respective face masks used were the B\&D Electromedical Fleximask (size small) and Koo full face mask system; fig. 5).

All the differences that can be observed in the figures are real differences with $p$-values that were so small as to be reported as equal to zero, even when the differences were as small as $0.22 \%$.

\section{Discussion}

The lung model enables the $V \mathrm{D} / V \mathrm{~T}$ fraction to be measured directly with great accuracy, with an average SE of 0.0018 or $0.5 \%$ of mean values. Being a laboratory study, the precision is such that even small unimportant differences noted in the figures are significantly different statistically speaking. All the differences that can be observed in the figures have p-values equal to zero as each reading was based upon 2,700 readings. The precision reflected by these statistics relates to the accuracy of the measuring equipment and the lung model arrangement. Variations between results may be affected to a much greater extent by application of the face mask to the mannequin or synchronisation of the ventilator.

$V \mathrm{D} / V \mathrm{~T}$ during spontaneous ventilation was increased by using face masks above $V$ D, phys from $32 \%$ to $42 \%$ of $V \mathrm{~T}$. The use of NIV modes such as bilevel and CPAP, with continuous pressure throughout the expiratory phase, reduced $V \mathrm{D} / V \mathrm{~T}$ to close to $V$ D,phys with most face masks. Pressure-assist and pressure-support ventilation without positive end-expiratory pressure (PEEP) decreased $V \mathrm{D} / V \mathrm{~T}$ to a lesser degree, from $42 \%$ to $39 \%$ of $V \mathrm{~T}$. Face masks with expiratory ports over the nasal bridge resulted in beneficial flow characteristics within the face mask and nasal cavity, which decreased $V \mathrm{D} / V \mathrm{~T}$ to less than $V \mathrm{D}$,phys from $42 \%$ to $28.5 \%$ of $V \mathrm{~T}$.

Not surprisingly, in spontaneous ventilation, $V \mathrm{D}$ increases the $V \mathrm{D}$,phys by $0.5(33.8) \%$. The correlation between the static volume measurements within face masks and the $V \mathrm{D}$ measurements was only 0.36 (fig. 2). This means that volumes within face masks bear little relation to the $V \mathrm{D}$ (fig. 4). Respironics

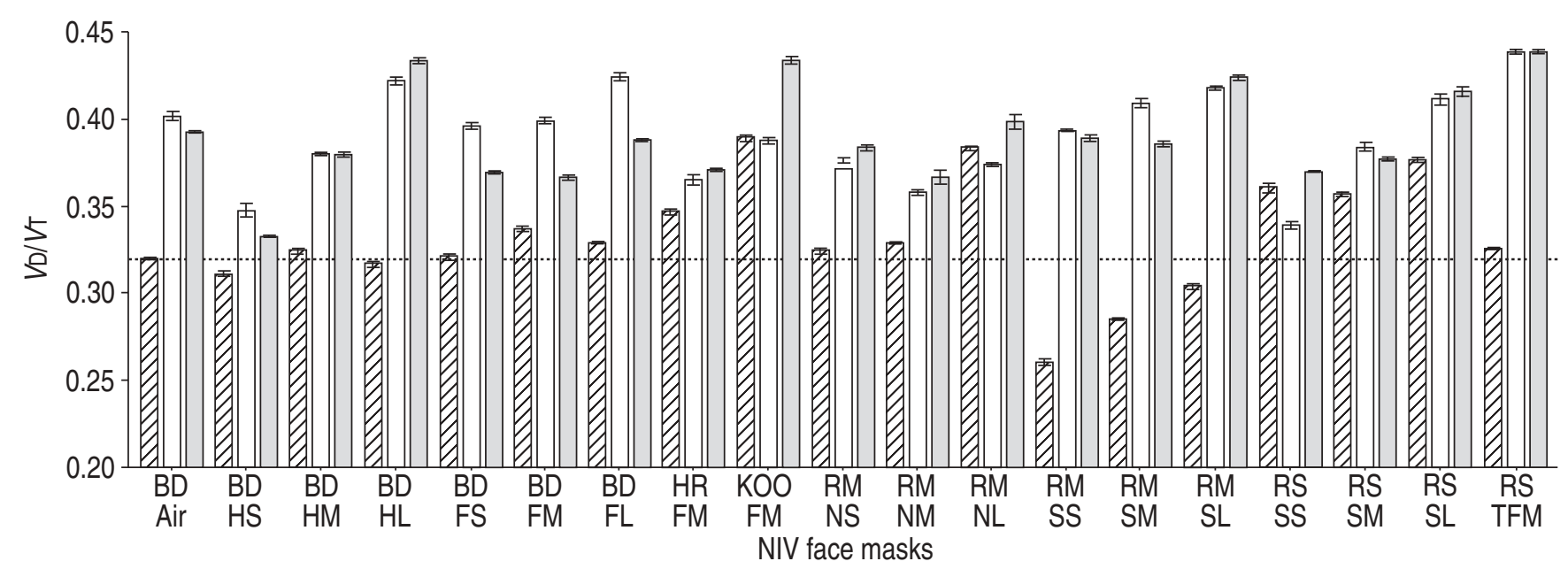

Fig. 7.-Total dynamic dead space $\left(V_{\mathrm{D}} / V_{\mathrm{T}}\right)$ in bilevel versus pressure-support and pressure-assist noninvasive ventilation (NIV) face masks. $\mathbb{Z}$ : Respironics BIPAP S/T; $\square$ : Breas 401; $:$ NIPPY 1 Dynamic total dead space $\left(V \mathrm{D} / V_{\mathrm{T}}\right)$. Refer to table 1 for abbreviations. 
total face mask is believed to have large dead space as it covers the whole face, and has a static dead space volume of $\sim 967 \mathrm{~mL}$. However, the present data show that even with spontaneous breathing, the $V \mathrm{D}$ is not much bigger than most other face masks. This unexpected result may be explained by the streaming effect of gas through the face mask.

The "elimination point" in a breathing system is the point beyond which exhaled alveolar gases are not returned to the alveolar compartment of the patient and, therefore, cannot contribute to $V \mathrm{D}$. For people breathing spontaneously under normal conditions, the elimination point is at the mouth. VD under these circumstances is then equal to $V \mathrm{D}$,phys, with $V \mathrm{D}$, ap equal to zero. During Bilevel and CPAP, because of the positive pressure during the expiratory phase, the elimination point moves within the face mask, closer to the patient so as to be almost identical to the usual point of elimination, at the mouth. Although there is an exhalation port in the patientbreathing system as well as peripherally located holes in some of the face masks, exhaled gases during Bilevel and CPAP, in contrast with spontaneous breathing, cannot be passing through the exhalation port of the breathing system, otherwise a greater $V \mathrm{D}$, ap would have been recorded in the study. This effectively makes the position of exhalation ports, expiratory valves that are located in any position other than somewhere attached to the face mask, redundant if an adequate positive pressure is maintained throughout the expiratory phase. This statement is not true if the expiratory pressure is equal to ambient pressure. This is illustrated in figure 7 by the observation that the same face masks under conditions of pressure-support and pressure-assist ventilation did not perform nearly as well. This finding has already been shown in the clinical setting $[4,5]$. In this study, it is both the flow during the expiratory phase and the flow pathway that was thought to lower $V \mathrm{D}$,ap.

In figure 5, face masks with exhaust ports or perforations over the nasal bridge worked best to decrease dead space. Exhaust ports at other sites were not nearly as good with ports located in the face mask over the cheeks performing slightly better than exhaust ports located at the maskconnector site. What may distinguish the ResMed Series 2 from the other face masks containing exhaust ports? Is it the fact that the perforations over the nasal bridge possibly result in asymmetrical gas flow within the face mask? If that were the reason, then the Hans Rudolph face mask with an exhaust port over one cheek would have performed notably better than the face masks with ports overlying both cheeks such as the Respironics total face mask. The differences noted here are too marginal to say that biased flow within the face mask is the explanation. To obtain a better understanding of the factors that influence $V \mathrm{D}$ in facemasks, fluid dynamic studies using engineering tools would be helpful in defining and guiding future designs of face masks.

By contrast, in spontaneous ventilation, it was surprising to find minimal effect on dead space with the use of exhaust ports on the face masks (Hans Rudolph full face mask, ResMed full face series 2, Respironics Spectrum and total face mask). It would be expected that exhaust ports in face masks would help expired flow to discharge by a beneficial flow stream and consequently reduce dead space [13]. However, if the pressure is low in the face masks during the expiratory phase, it would appear to limit the effectiveness of gas elimination via the holes in the face mask, thereby not providing the flow stream characteristics that are seen at higher pressures.

Since one of the aims of this study was to demonstrate the effects of different NIV ventilation modes upon $V D$, ap, the most commonly used NIV ventilation modes were examined: Bilevel, CPAP, pressure-support ventilation, and pressure-assist ventilation (figs 5-7). It was shown that with all face masks,
$V \mathrm{D} / V \mathrm{~T}$ was reduced considerably when using Bilevel or CPAP (from 0.42 to 0.33 and 0.34 , respectively) and pressuresupport or pressure-assist ventilation (0.39). It is to be noted that the $V \mathrm{D} / V \mathrm{~T}$ fraction is reduced more in the case of Bilevel and CPAP than pressure-support and pressure-assist ventilation. What Bilevel and CPAP have in common and in contrast with pressure-support and pressure-assist ventilation, is constant pressure throughout the expiratory phase. The reason this decreases dead space is that the positive pressure during expiration forces exhaled gas out of the main stream, thus moving the elimination point nearer to the patient. In other words, the effect of fresh gas flow during expiration is directly related to good flushing of the face mask.

There was no important difference in the $V \mathrm{D} / V \mathrm{~T}$ fraction between Bilevel and CPAP, nor was there a difference between the different makes of Bilevel ventilators. From the findings it is unknown whether other ventilator parameters, such as inspiratory flow pattern, inspiratory and expiratory triggering or response time are important influences of the $V \mathrm{D} / V \mathrm{~T}$ fraction. Although it is likely they have little influence, further study is needed to describe the detailed effects of ventilator trigger characteristics.

Comparing pressure-support with pressure-assist ventilation, no difference was found between $V \mathrm{D}$ measurements. As the expiratory phase is the key to determining $V \mathrm{D}$, it is not surprising that there is no difference between these modes as there is zero flow from the ventilator side during expiration in both.

The differences between the masks were minor. The biggest $V D$ measured was the Koo full face mask system and the smallest was the ResMed full face series 2 during Bilevel. This was recorded as less than the $V \mathrm{D}$,phys. This may be explained from the flow characteristics within the mask design. The ResMed full face series 2 face mask has openings at the level of the bridge of the nose. During CPAP and Bilevel, flow circulation within the face mask of the current model would appear to cause air to circulate via the mouth opening and nasal cavity, decreasing anatomical dead space by shifting the elimination point to the level of the nasopharynx.

From figure 5 it may be observed that similar results occurred using the B\&D Electromedical small and medium fleximask face masks with ResMed Sullivan VPAP S/T ventilator as with the ResMed series 2 using Bilevel or CPAP modes. To explain this finding, it must be postulated that similar face mask flushing mechanisms that involved the nasal cavity must have been in operation. That leads to the question of why did it not occur with other Bilevel ventilators? There is always the possibility that when using the B\&D Electromedical small and medium fleximask face masks, the ability to hold a reliable seal at the nose of those particular design and sizes of face mask may not have been as good on the mannequin as on a real face. So, in effect, a leak at the nose could conceivably have produced the same effect as the dedicated nasal exhaust ports on the ResMed series 2 face masks. The results were obtained blind, so there is no way of knowing what the real mechanism is in this instance.

The limitation of the model was that leaks were sometimes present between the mannequin head and face masks. Leaks were minimised by applying masks tightly but without any shape and volume deformity. However, with some masks there may be minor leaks due to fitting problems. Since ventilators compensated for minor leaks and the compared ventilation modes were constant pressure-based, any errors are probably unimportant. The likely effect of any such error is to be biased in the direction of decreasing dead space as it has been shown that poorly fitting masks decrease $V D$ [13]. A second problem encountered was that some ventilators failed to trigger with every breath. The NIPPY 1 trigger decreased 
to $66 \%$, while with BIPAP S/T 30 and ResMed Sullivan S/T triggering were $100 \%$ with all masks.

To conclude, it has been demonstrated that the interface between noninvasive ventilators and patients are probably more important than the mode of ventilation [14]. Of the interfaces, it also showed that face masks produced the best blood-gas results. A very important component, namely $V \mathrm{D}$, has been studied within this most frequently used interface. NIV face masks increase the $V \mathrm{D}$, phys by $0.5(33.82) \%$ in spontaneous ventilation. There is a poor relationship between the static volume within face masks and the $V \mathrm{D}$, ap measurements. Although, when using ventilators, the differences between face masks are accentuated and the $V D$ decreases in all of them. The amount that $V \mathrm{D}$ decreases depends on both ventilation mode and the face mask itself. Bilevel and CPAP modes are more effective in decreasing the $V \mathrm{D}$ than pressuresupport and pressure-assist ventilation (without PEEP in the case of NIV ventilators) because positive pressure is applied throughout the expiratory phase.

Exhaust ports in the nasal bridge of face masks encourage a beneficial flow path that decreases dead space, but only if used in combination with ventilation modes that employ positive pressure throughout the expiratory phase.

\begin{abstract}
Acknowledgements. The authors would like to thank D. Light for technical support, and Lane Fox Unit of St Thomas' Hospital for kindly lending them the noninvasive ventilators and $\mathbf{M}$. Latham of St James's University Hospital, Leeds, UK, who lent them the face masks.
\end{abstract}

\section{References}

1. Marsh MJ, Ingram D, Milner AD. The effect of instrumental dead space on measurement of breathing pattern and pulmonary mechanics in the newborn. Pediatr Pulmonol 1993; 16: $316-322$.
2. Hoskins SC, Patz JD. Effects of small and large facemasks and translarnyngeal and tracheostomy intubation on ventilation, upper-airway dead space, and arterial blood gases. Am $J$ Vet Res 1986; 47: 945-948.

3. Ferguson GT, Gilmartin M. $\mathrm{CO}_{2}$ rebreathing during BIPAP ventilatory assistance. Am J Respir Crit Care Med 1995; 151: 1126-1135.

4. Lofaso F, Brochard L, Touchard D, Hang T, Harf A, Isabey D. Evaluation of carbon dioxide rebreathing during pressure support ventilation with airway management system (BIPAP) devices. Chest 1995; 108: 772-778.

5. Chen R, Zhang X, He G. Modification of facial mask on the dead space effect in non-invasive mask ventilation. Zhonghua Jie He He Hu Xi Za Zhi 2000; 23: 734-736.

6. Fletcher R, Jonson B, Cumming G, Brew J. The concept of deadspace with special reference to the single breath test for carbon dioxide. Br J Anaesth 1981; 53: 77-87.

7. Vavra J, Smejkal V. Ventilation of an additional dead space. Physiologia Bohemoslovaca 1990; 39: 443-447.

8. Whitwam JG. Functional deadspace during high frequency ventilation. Eur J Anaesth 1984; 1: 7-9.

9. Arborelius M, Rosberg HE, Wiberg R. Multiple breath nitrogen dead space. Clin Physiol 1988; 8: 561-576.

10. Williams EM, Gavaghan DJ, Oakley PA, et al. Measurement of dead-space in a model lung using an oscillating inspired argon signal. Acta Anaesthesiol Scand 1994; 38: 126129.

11. Williams EM, Hamilton RM, Sutton L, Viale JP, Hahn CEW. Alveolar and dead space volume measured by oscillations of inspired oxygen in awake adults. Am J Respir Crit Care Med 1997; 156: 1834-1839.

12. Miller DM. Breathing systems for use in anaesthesia. $\mathrm{Br}$ $J$ Anaesth 1988; 60: 555-564.

13. Miller DM. Paediatric breathing systems: their use and misuse in South Africa. SAJAA 1998; 1: 28-34.

14. Navalesi P, Fanfulla F, Frigerio PRT, Gregoretti C, Nava S. Physiologic evaluation of noninvasive mechanical ventilation delivered with three types of masks in patients with chronic hypercapnic respiratory failure. Crit Care Med 2000; 28: $1785-1790$. 